\title{
Manganese abundances in cluster and field stars
}

\author{
Jennifer S. Sobeck ${ }^{1}$, Jennifer Simmerer, ${ }^{1}$ Inese I. Ivans, ${ }^{2}$ \\ Christopher Sneden, ${ }^{1}$ Jon P. Fulbright, ${ }^{3}$ and Robert P. Kraft ${ }^{4}$ \\ ${ }^{1}$ Department of Astronomy, University of Texas at Austin, Austin, TX 78712, USA \\ email: jsobeck@mail.utexas.edu, jensim@astro.as.utexas.edu, chris@verdi.as.utexas.edu \\ ${ }^{2}$ Department of Astronomy, California Institute of Technology, Pasadena, CA 91125, USA \\ email: iii@astro.caltech.edu \\ ${ }^{3}$ Carnegie Observatories, Pasadena, CA 91101, USA \\ email: jfulb@ociw.edu \\ ${ }^{4} \mathrm{UCO} /$ Lick Observatory, Department of Astronomy and Astrophysics, University of \\ California, Santa Cruz, CA 95064, USA \\ email: kraft@ucolick.org
}

\begin{abstract}
We have derived Mn abundances for more than 200 stars in nineteen globular clusters. In addition, Mn abundance determinations have been made for a comparable number of halo field stars possessing an overlapping range of metallicities and stellar parameters. The primary data set was comprised of high resolution spectra previously-acquired at the McDonald, Lick and Keck Observatories. Additional data were obtained from several other investigators. Data were analyzed using synthetic spectra of the $6000 \AA$ Mn I triplet. Hyperfine structure parameters were included in the synthetic spectra computations. Our analysis shows that over the metallicity range $-0.7>[\mathrm{Fe} / \mathrm{H}]-2.7$ field stars have a mean relative abundance of $\langle[\mathrm{Mn} / \mathrm{Fe}]\rangle=-0.36$ identical to that of the nineteen globular clusters $\langle[M n / F e]\rangle=-0.36$. Our Mn abundance results viewed in conjunction with the globular cluster $\mathrm{Cu}$ abundances of Simmerer et al. (2003) suggest the following possibilities: one, the production of these elements is highly metallicitydependent or two, these elements were manufactured in the Galactic halo prior to formation of present-day globular clusters.
\end{abstract}

Keywords. Galaxy: abundances, Galaxy: halo, Galaxy: globular clusters, stars: abundances

\section{Introduction}

Elemental abundance trends with overall metallicity contain vital clues to the formation and evolution of the Galaxy. Comparison of stellar populations is necessary in order to establish trends in abundance for the various element groups. It has been shown that the metallicity ranges of globular cluster and halo field stars overlap (e.g. Laird et al. 1988). Hence, these metal-poor stars provide insight into the earliest stages of Galactic chemical evolution.

The only elements to display significant differences in abundance between halo field and globular cluster stars are those of the proton-capture group (McWilliam 1997). The abundances for the vast majority of other elements are virtually the same in the two stellar populations (Gratton et al. 2004). Similarly, Fe-peak elements exhibit equivalent abundance levels in stars of globular clusters and the halo field. In fact, a solar abundance is observed for most members of the Fe-peak. Notable exceptions to this are Copper and Manganese. In the metallicity range of $-2.5<[\mathrm{Fe} / \mathrm{H}]<-1.0$, Mishenina et al. (2002, and references therein) detected a distinct subsolar abundance of $\mathrm{Cu}$ in halo field giants. Simmerer et al. (2003) found an analogous $\mathrm{Cu}$ deficiency in globular clusters. 


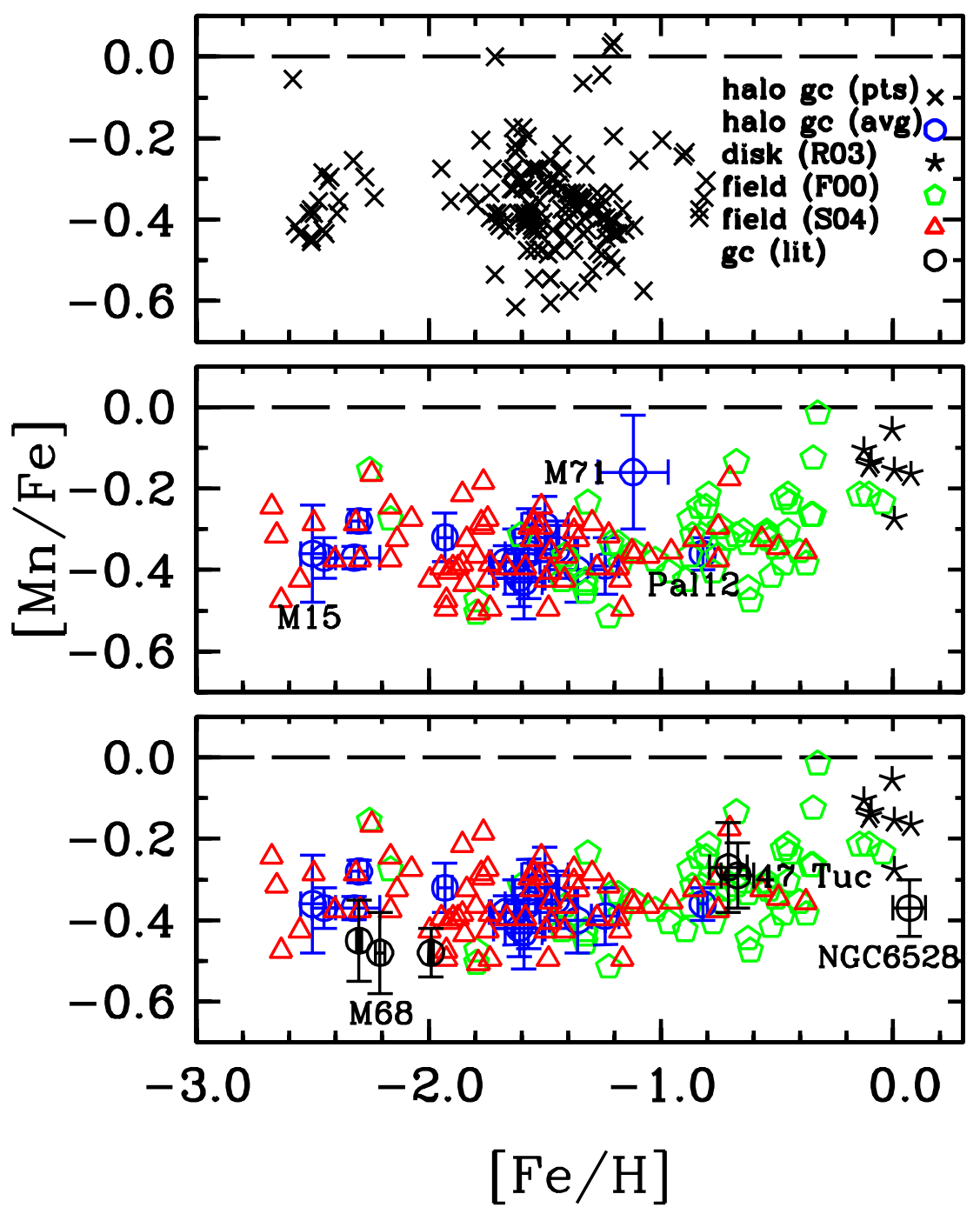

Figure 1. Correlation of $[\mathrm{Mn} / \mathrm{Fe}]$ with $[\mathrm{Fe} / \mathrm{H}]$ for different stellar samples. The upper panel presents all abundances for stars of the globular cluster data sample. The middle panel shows the average $[\mathrm{Mn} / \mathrm{Fe}]$ and $[\mathrm{Fe} / \mathrm{H}]$ values for each cluster (with associated error bars) as well as field star data. The inclusion of literature values occurs in the bottom panel. The figure is taken from Sobeck et al. (2005).

Wallerstein (1962) was among the first to observe a subsolar Mn in metal-poor field stars. With the use of hyperfine structure data, Gratton et al. (1989) and McWilliam (1997) were able to confirm these observations and derive more accurate Mn abundance ratios. The shortage of available transitions in the red portion of the visible spectrum and the lack of adequate hyperfine structure computations have hampered Mn abundance analyses. Consequently, a paucity of Mn data (particularly for globular clusters) exists in the literature. This lack of data necessitated a thorough and systematic study; the results of which are presented here. The main goal of the study is the determination of Mn abundance in globular clusters and then the subsequent comparison to the halo field in order to develop a trend in Mn abundance. 


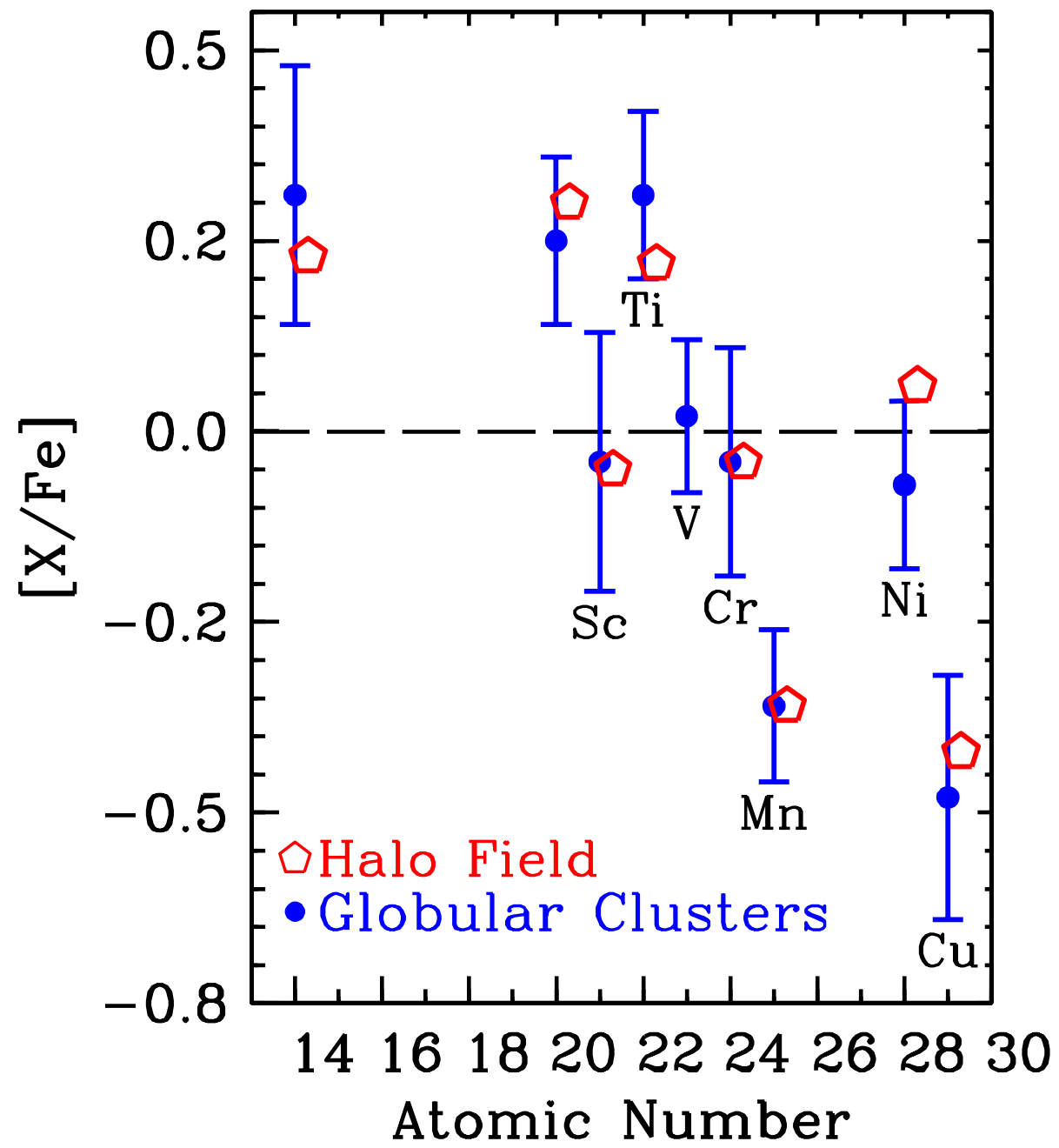

Figure 2. The average abundance ratios are given for some of the Fe-peak and $\alpha$-elements. Notice that for most elements the average values for globular cluster and halo field stars are virtually equal to one another.

\section{Observations and Data Analysis}

For abundance determinations, spectroscopic and equivalent width data were acquired from many sources. The initial data set was taken from spectra previously gathered from the Lick-Texas series of investigations into globular cluster chemistry (hereafter labeled as LTG: Kraft et al. (2004) and references therein). Sample size varies from as little as two to as many as twenty-three stars for the globular cluster data. The two field star surveys each have a sample size in excess of 80 stars. Stars in the field data sample are of a variety of evolutionary states whereas the bulk of the globular cluster data are luminous red giants. Three telescope facilities were utilized for these observations: Keck I (HIRES Spectrograph), Lick (Hamilton Spectrograph), and McDonald (2d-coudé Spectrograph). The remainder of the globular and open cluster spectra were obtained from several external sources. These data were collected with the VLT, APO, CTIO, and 
Keck I telescopes. As a whole, the data set had high spectral resolution with $R>24,000$ and high signal-to-noise $(\mathrm{S} / \mathrm{N})$ values.

A standard abundance determination (e. g. Simmerer et al. 2004) ensued in which three spectral features of Mn (6013 $\AA, 6016 \AA$, and $6021 \AA$ ) were selected for analysis. These features are the only strong transitions of $\mathrm{Mn}$ in the yellow-red wavelength regime (saturation is rarely a concern). To achieve the most correct measurement of Mn abundance, hyperfine structure was taken into consideration. To obtain a local iron abundance, two nearby Fe features $(6024 \AA$ and $6027 \AA)$ were chosen. By doing this, continuum and continuous opacity effects were eliminated. Also, there is a direct measure of the behavior of $\mathrm{Mn}$ relative to $\mathrm{Fe}$ as the transitions lie on the same order. MARCS (Gustaffson et al. 1975) and ATLAS Kurucz (1993) codes were employed to generate model atmospheres. The LTE line analysis and spectral synthesis code MOOG (Sneden 1973) was utilized to extract $[\mathrm{Mn} / \mathrm{Fe}]$ values. The spectral synthesis technique allows for discernment of fine changes in abundance, and it can accurately account for hyperfine structure.

\section{Results}

Figure 1 displays $[\mathrm{Mn} / \mathrm{Fe}]$ as a function of $[\mathrm{Fe} / \mathrm{H}]$ for both field and globular cluster stars. The upper panel features values for the individual stars of the globular clusters. The data present a mostly uniform $[M n / F e]$ with few outliers. For the halo globular clusters, Pal 12 and M15 represent the metal-rich and metal-poor extremes respectively. The middle panel shows the average $[M n / F e]$ values for the globular clusters and includes the data from the field star surveys. Intra-cluster Mn abundance variation is small (due primarily to observational uncertainty). Error in $\mathrm{Fe}$ is somewhat larger and may be attributed to the lack of features employed in the abundance determination. Table 1 lists the average abundance ratios for the LTG globular clusters. As compared to other clusters, M71 gave an unexpectedly high Mn abundance. M71 data acquisition occurred prior to instrument update. Utilizing high-quality Keck I data, Ramirez \& Cohen (2002) report $\langle[M n / F e]\rangle=-0.27$ for this cluster; a result much more in line with that of other globular clusters. And as shown in the bottom panel, literature data points for five globular clusters concur with the results of this study.

For the entire data set in the metallicity range $-2.7<[\mathrm{Fe} / \mathrm{H}]<-0.7,<[\mathrm{Mn} / \mathrm{Fe}]>=$ $-0.36 \pm 0.01$ for globular clusters and $<[M n / F e]>=-0.36 \pm 0.01$ for the halo field. Error analysis consisted of varying both the stellar atmospheric and spectral fit parameters to determine the effect on fit quality. Thus, these Mn data indicate that there is no statistically significant difference between the halo field and globular cluster stars. As $[\mathrm{Fe} / \mathrm{H}]$ increases toward zero, so correspondingly does $[\mathrm{Mn} / \mathrm{Fe}]$. The data does not clearly indicate that at solar metallicity a solar $\mathrm{Mn}$ abundance is achieved (perhaps a slight offset is present). For the bulge globular cluster NGC 6528, Carretta et al. (2001) find a rather low $\mathrm{Mn}$ abundance at high metallicity with $<[\mathrm{Fe} / \mathrm{H}]=0.07>$ and $<$ $[M n / F e]=-0.37>$. At higher metallicites, the trend in Mn abundance is not apparent and further study is required.

\section{Conclusions and Discussion}

We have employed spectral synthesis to extract the Mn abundance ratio for hundreds of globular cluster, halo field, and open cluster stars. In the metallicity range $-0.7>$ $[\mathrm{Fe} / \mathrm{H}]>-2.7$, the $\mathrm{Mn}$ abundance ratio is found to be the exact same in stars of both globular clusters and the halo field. With respect to $\mathrm{Mn}$, the two stellar populations are indistinguishable. 
Table 1. Mean Abundances for the LTG Clusters

\begin{tabular}{lccccc}
\hline Cluster & $\langle\mathrm{Fe} / \mathrm{H}]\rangle$ & $\sigma$ & $\langle\mathrm{Mn} / \mathrm{Fe}]\rangle$ & $\sigma$ & $N_{\text {stars }}$ \\
\hline M10 (NGC 6254) & -1.63 & 0.11 & -0.38 & 0.08 & 12 \\
M4 (NGC 6121) & -1.24 & 0.06 & -0.39 & 0.07 & 20 \\
Pal 5 & -1.50 & 0.10 & -0.29 & 0.07 & 4 \\
M5 (NGC 5904) & -1.36 & 0.12 & -0.40 & 0.08 & 23 \\
NGC 7006 & -1.61 & 0.11 & -0.42 & 0.07 & 6 \\
M15 (NGC 7078) & -2.50 & 0.05 & -0.36 & 0.12 & 11 \\
M3 (NGC 5272) & -1.59 & 0.08 & -0.43 & 0.09 & 20 \\
M71 (NGC 6838) & -1.12 & 0.15 & -0.16 & 0.14 & 10 \\
M13 (NGC 6205) & -1.67 & 0.06 & -0.38 & 0.04 & 17 \\
M92 (NGC 6341) & -2.45 & 0.05 & -0.37 & 0.05 & 4 \\
\hline
\end{tabular}

Figure 2 displays a plot of elemental abundance ratio as a function of atomic number. For the members of the Fe-peak, the abundances for halo field stars are essentially equivalent to those of globular cluster stars. The data in Figure 2 also serves as further verification of the even-odd effect. As shown, there are sub-solar levels of Mn and other odd Z-numbered elements. The production of these elements is dependent upon neutron excess which is deficient for metal-poor stars (e. g. Nakamura et al. 1999).

In the region of $-0.7>[F e / H]>-2.7$, a plateau in $[M n / F e]$ is seen. The singlevalued $[M n / F e]$ ratio is due to the lack of variability in contributing progenitors. In effect, the production of $\mathrm{Mn}$ and Fe is due to the same segment of the IMF.

A continuation of Mn investigations into metal-rich regions should occur with emphasis placed on Bulge Globular Clusters. In addition, a verification of the Mn abundance trend in the infrared wavelength regime should take place with the logical extension to metalrich target stars.

\section{Acknowledgements}

Ongoing support from the NSF, currently through grants AST-0307495 to CS and AST-0098453 to RPK, is gratefully acknowledged. Research for III is currently supported by NASA through a Hubble Fellowship grant HST-HF-0115101-A from the STSI. JSS gratefully acknowledges enlightening discussions with P. Hoeflich and E. Carretta.

\section{References}

Carretta, E., Cohen, J. G., Gratton, R. G., \& Behr, B. B. 2001, AJ 122, 1469

Cohen, J.G. 2004, $A J$ 127, 1545

Fulbright, J.P. 2000, $A J$ 120, 1841

Gratton, R.G. 1989, A\&A 208, 171

Gratton, R.G., Sneden, C., \& Carretta, E. 2004, ARA\&A 42, 385

Gustaffson, B., Bell, R.A., Eriksson, K., \& Nordlund, A. 1975, A\& A 42, 407

Kraft, R.P. 2004, MmSAI 75, 295

Kurucz, R.L. 1993, Kurucz CD-ROM 13, ATLAS 9 (Cambridge:SAO)

Laird, J.B., Rupen, M.P., Carney, B.W., \& Latham, D.W. 1988, AJ 96, 1908

McWilliam, A. 1997, ARAA 35, 503

Mishenina, T.V., Kovtyukh, V.V., Soubrain, C., Travaglio, C., \& Busso, M. 2002, A $\& A$ 396, 189

Nakamura, T., Umeda, H., Nomoto, K., Thielman, F.K., \& Burrows, A. 1999, ApJ 517, 193

Ramirez, S.V., \& Cohen, J.G. 2002, ApJ 123, 3277 
Simmerer, J.A., Sneden, C, Ivans, I.I., Kraft, R.P., Shetrone, M.D., \& Smith, V.V. 2003, AJ 125, 2018

Simmerer, J.A., Sneden, C., Cowan, J.J., Collier, J., Woolf, V.M., \& Lawler, J.E. 2004, ApJ 617, 1091

Sobeck, J.S. 2005, in press

Sneden, C. 1973, ApJ 184, 839

Wallerstein, G. 1962, ApJS 6, 407 